\title{
Contes de la femme convoitée et calomniée. À propos de la classification de contes-nouvelles
}

\author{
Camiño Noia Campos \\ Universidade de Vigo \\ cnoia@uvigo.es
}

\begin{abstract}
RÉSUMÉ
Sous le terme 'Romantic' ou 'Réalistic' la section « Romantic Folktales (Novelle) » inclute des contes de nature différente; quelques-uns ayant une forte coïncidence avec des types des «Magic Tales » et suivant la variation des personnages, ils coïncident aussi avec les "Religious Tales ». Je parlerai des caractéristiques des contes rassemblés dans la section "Romantic Folktales », de certains aspects spécifiques et des éventuels traits communs qu'ils ont avec des contes d'autres sections : en spécial avec celle des merveilleux (Magic Tales). Dans la seconde partie de cet article, je comparerai plusieurs versions de contes merveilleux et romanesques de différentes régions de la Péninsule Ibérique traitant du thème de la chasteté [K2II2] et de la fidélité [K2IIO.I] de la femme (7I2-Crescencia, 88I-Oft-Prouved Fidelity et 883A-The Innocent Slandered Maiden) dans les versions orales et les versions écrites rassemblées dans des textes écrits depuis le Moyen-Âge jusqu'au commencement du XX $x^{\text {ìme }}$ siècle.
\end{abstract}

MOTS CLÉS

femme calomniée; innocente; nouvelle; récit de magie; ATU 7I2; ATU 883A

\section{RESUM}

Sota l'adjectiu «romàntic» o «realista» la secció «Romantic Folktales» (Novelle) inclou rondalles de diferent naturalesa; algunes d'elles tenen una forta coincidència amb els tipus dels «Magic Tales» i, seguint la variació dels personatges, coincideixen també amb els «Religious Tales». En aquest article parlaré de les característiques de les rondalles incloses en la secció "Romantic Folktales», de certs aspectes específics $i$ d'altres trets eventuals que tenen en comú amb rondalles d'altres seccions: especialment amb les rondalles meravelloses (Magic Tales). En la segona part d'aquest article, compararé diverses versions de rondalles de diferents parts de la península Ibèrica que tracten el tema de la castedat [K2II2] i de la fidelitat [K2IIO.I] de les dones (7I2-Crescencia, 88I-Oft-Prouved Fidelity $i 883$ A-The Innocent Slandered Maiden) en les versions orals i escrites recollides en textos escrits des de l'edat mitjana fins a principis del segle XX.

PARAULES CLAU

dona calumniada; innocent; novel-lesc; rondalla meravellosa; ATU 7I2; ATU 883A 


\begin{abstract}
Under the adjectives 'romantic' or realistic, the Romantic Folktales section includes different types of tales. Some of them have strong similarities to certain types of Magic Tale and, if certain changes are made to the protagonists, to Religious Tales. This article analyses the characteristics of the tales included in the Romantic Folktales section and examines certain specific features that they have in common with other sections, in particular Magic Tales. The second part of the article compares various versions of tales from different linguistic areas of the Iberian Peninsula that deal with the themes of chastity [K2II2] fidelity [K2IIO.I] women (7I2-Crescentia, 88I-Oft-Proved Fidelity and $883 A$-The Innocent Slandered Maiden). The tales are in the form of oral and written versions collected from written texts dating from the Middle Ages up to the beginning of the 2 oth century.
\end{abstract}

\title{
KeYwORDS
}

Innocent Slandered Maiden; Magic Tales; ATU 7I2; ATU 883A

REBUT: I6.02.2OI4 | ACCEPTAT: I3.05.2OI4 


\section{Introduction}

Quand, en I9Io, Antti Aarne propose le premier classement des contes de tradition orale, Verzeichnis der Märchentypen, il établit seulement trois sections : I. «Tiermärchen» (contes animaux), II. «Eigentliche Märchen » (contes proprement dits / réalistes) et III. "Schwänke » (contes facétieux). Mais, étant donné que les contes groupés sous le titre «Eigentliche Märchen » étaient de différente nature, il essaya d'organiser cet ensemble divers en quatre sous-groupes: A. «Zaubermärchen » (contes merveilleux), B. «Legendenartige Märchen » (contes religieux), C. « Novellenartige Märchen » (contes-nouvelles) et D. " Märchen vom dummen Teufel » (histoires d'ogres stupides). Quatre groupes de contes qu'Antti Aarne organise selon des critères différents: Ceux qui ont des éléments magiques; ceux qui traitent d'un thème ou de personnages religieux (avec des éléments issus du merveilleux chrétien); ceux qui racontent des histoires d'aventures diverses: amoureuses, facétieuses sur la relation conjugale, didactiques..., où l'homme agit sans l'aide d'adjuvants ou d'éléments surnaturels et, finalement, ceux de héros vaillants défiant le diable, des ogres ou des géants.

Le travail de classification d'Aarne a été fondamental pour les folkloristes qui, grâce à ce classement scientifique, ont pu disposer d'une méthode pour appréhender les contes populaires de manière systématique et sur laquelle continuer à réfléchir. Il ne faut pas oublier qu'en I9Io ce premier classement faisait suite à un siècle de collectes et d'analyses comparatives. Mais, comme tous les premiers apports scientifiques, celui d'Aarne était une approche provisoire, en attendant d'en trouver une autre plus appropriée pour intégrer des contes que l'on ne pouvait insérer dans les trois groupes proposés.

La mort prématurée, en I925, du folkloriste finlandais a certainement limité les initiatives de Stith Thompson en tant que continuateur de son projet. Dans les éditions du catalogue international, The Types of Folktales, parues en 1928 et en I96I sous le nom des deux folkloristes, l'américain a respecté la classification d'Aarne et traduit simplement le titre du sous-groupe " Novellenartige Märchen » comme « Novelle (Romantic Tales) », en plus d'avoir intégré de nouveaux types et ajouté la section des «Formula Tales ». Dans la révision qu'il en publie en 2004, Hans-Jörg Uther a fait d'importants changements. Il a réduit le nombre de soustypes et intégré nombre de références issues des catalogues régionaux. Il a remplacé l'adjectif 'Romantic' de Thompson, par celui de 'Realistic'; sans doute, avec l'intention de regrouper les divers thèmes qu'il y a dans cette section, et il a surtout modifié l'organisation de l'ensemble des contes réalistes en rendant des sections indépendants les quatre sous-groupes: "Tales of Magic », « Religious Tales », « Realistic Tales (Novelle) » et « Tales of the Stupid Ogre (Giant, Devil) » qui figuraient sous la section II, « Ordinary Folk-Tales ».

Malgré tout, sous la dénomination de «Realistic Tales » on trouve encore des contes de nature différente : des histoires de princesses qui se distraient en proposant des devinettes que résolvent des jeunes gens pauvres mais astucieux, des rois qui tombent amoureux de jeunes paysannes charmantes et des contes au développement varié présentant des héros modestes qui réunissent par hasard une grande fortune. Et on peut trouver aussi des histoires réalistes enrichies par des épisodes de légendes religieuses ou mythologiques, comme, la présence d'un monstre difficile à vaincre, des apparitions magiques ou miraculeuses grâce à l'in- 
tervention d'un saint ou de la Sainte Vierge, dont le thème est proche des « Magic Tales » ou des « Religious Tales ». En référence à la section « Realistic Tales », Marie-Louise Tenèze dans l'Introduction au catalogue français des Contes-nouvelles (Delarue-Tenèze 2000: Io) montre déjà que « la délimitation de cette section du Catalogue $[\mathrm{AT}]$ n'est rien moins qu'évidente », donc elle rassemble des contes de plusieurs sortes.

S'il est vrai que dans la narration orale les conteurs agissent en liberté lorsqu'ils racontent une histoire, et s'ils peuvent supprimer ou incorporer des épisodes et des éléments magiques, c'est toujours en raison de leurs récepteurs et du contexte social, et dans certaines limites. En ce sens, l'utilisation des contes dans la prédication de l'Église, ainsi que le passage de l'oral à l'écrit ou de l'écrit à l'oral depuis le Moyen Âge, ont été fondamentaux pour l'intégration des éléments religieux (magiques) dans les contes réalistes.

Il est bien connu qu'un grand nombre d'histoires contenues dans les Mille et une nuits, le Decaméron, le Livre de Patronio, et dans d'autres collections de contes, sont plutôt des histoires qui sont passées d'une source orale à une savante et ont circulé dans les cultures occidentales par le truchement de l'écrit et de l'oralité. Et, même si elles sont d'origine orale et «se propagent en des versions différentes, obéissant à des règles établies par la tradition», tel que le souligne Pinto Correia (I992: II6), l'influence savante est déterminante. Bien que très populaires à l'oral, c'est la forme écrite de la « Nouvelle» qui, en général, va les caractériser. Les contes qui y sont classés ont généralement une expression textuelle plus élaborée que celle des contes traditionnels, d'autant plus qu'ils sont composés de plusieurs épisodes déroulant des histoires complexes, coïncidant, sans doute, avec quelques contes merveilleux. Nous devrons donc souligner leur étroite relation avec les récits écrits comme un élément de grande importance pour déterminer l'attribution des contes au groupe de « Realistic Tales».

\section{Types 712 et $883 \mathrm{~A}$ dans les textes écrits et dans les contes oraux}

Pour illustrer l'étroite relation qu'il y a entre contes qui appartiennent à l'un et à l'autre groupes de l'ATU, on comparera les motifs des deux types suivants : le conte-type 7I2 dans la catégorie des «Magic Tales » et le conte-type 883A relevant des «Realistic Tales». On analysera les versions de ces deux types de contes oraux recueillis dans les différentes aires linguistiques de la Péninsule Ibérique, ainsi que leur mode de circulation dans la littérature écrite et dans l'oralité. Ces deux contes-types racontent l'histoire de la femme convoitée, calomniée et abandonnée dans un bois (motifs K2IIO.I, K22II.I et K2II2).

D'origine orientale, comme Adolphe Mussafia (I866) l'a montré au XIX ${ }^{\text {ème }}$ siècle et comme d'autres folkloristes l'ont corroboré (Uther 2oII, Part. I : 386), l'histoire de la femme chaste accusée d'adultère est un thème universel, appartenant à la tradition, tant écrite qu'orale, de nombreuses cultures. La première référence chronologique du thème peut se trouver dans l'épisode de «Susanne et les vieillards » du «Livre de Daniel » (vi ${ }^{\text {ème }}$ siècle A. C.) de l'Ancien Testament. Elle se retrouve également dans l'épopée hindoue Le Rãmãyana (II ${ }^{\text {ème }}$ siècle) et dans l'« Histoire du Cadi Juif et sa vertueuse épouse » des Mille et Une nuits (nuits 280 et 28I). 
L'histoire d'innocentes femmes convoitées et injuriées a été romancée sous des traits légendaires dans des chansons de geste européennes, attribuées à Béatrice, l'épouse de Charlemagne, ainsi qu'à sa mère, Berthe, comme le démontrent les nombreux récits écrits du Moyen âge jusqu'au XVIII ${ }^{\text {ème }}$ siècle, ainsi que des versions orales qui circulent ( $c f$. Frenzel [I976] I980; Uther, EM, 3: I67-I7I).

L'histoire raconte qu'en l'absence de son mari (père) qui part pour un long voyage, sa belle épouse (fille) reçoit des propositions amoureuses de la part d'un domestique (son beau-frère ou son oncle) qui s'est épris d'elle. Comme elle le repousse, le séducteur l'accuse d'adultère. Quand le mari (père) revient, il ordonne de la tuer dans la forêt. Elle est sauvée de la mort, mais devra vivre plusieurs aventures jusqu'à ce qu'elle retrouve son mari (père) et démontre son innocence.

On trouve ce récit dès le XII ${ }^{\text {ème }}$ siècle, dans la Gesta regum Anglorum (II27) de l'anglais William de Malmesbury, dont le protagoniste est Gunilda (ou Cunigunda), femme de l'empereur allemand Henrik; dans ce récit la Vierge Marie est l'adjuvant magique qui aide l'héroïne et la sauve de la mort. L'histoire est aussi dans la narration en vers Kaiserchronik (II35-II5O), où l'impératrice romaine Crescencia occupe la place de Gunilda (Frenzel I980: 127).

La bibliothèque du monastère de L'Escorial, près de Madrid, conserve l'ouvrage des Cantigas de Santa María que le roi espagnol Alphonse X a fait écrire au XIII ${ }^{\mathrm{èm}}$ siècle, en langue galicienne et en vers, dont la «Cantiga V » intitulée «Esta é como Santa María ajudou a Emperatriz de Roma a sofre-las grandes coitas per que passou ", ${ }^{\text {I }}$ qui raconte l'histoire de l'impératrice de Rome injustement accusée d'adultère. Le roi pouvait avoir trouvé le récit dans n'importe quel texte circulant à l'époque, être dans la Gesta regum Anglorum, parce qu'il est écrit dans l'introduction de la cantiga «E desto vos quer' eu ora contar, segund' a letra diz ». ${ }^{2}$

Des histoires semblables se trouvent dès le XIII ${ }^{\text {ème }}$ siècle dans d'autres collections des miracles de la Sainte Vierge ${ }^{3}$ et dans des exempla médiévaux, où, parfois, Saint Pierre occupe la place de la Vierge (Frenzel I980: I26).

Du premier quart du XIII ${ }^{\mathrm{ème}}$ siècle est daté le roman anglais en vers La Bone Florence of Rome, qui a un rapport avec la légende de Berthe, mère de Charlemagne, et qui a influencé l'Histoire de la Reine Sibille et Macario dans la première moitié du XIV ${ }^{\mathrm{e}}$ siècle ainsi que la Gesta Romanorum (I342), où l'on trouve un récit sur le même thème.

Ce thème, qui a eu une grande vitalité au Moyen Âge, s'est diffusé en France et en Allemagne, et a découlé des versions différentes venues de divers pays européens, pleines de combinaisons et de motifs variés et dont les héroïnes se nomment Crescencia mais aussi Béatrice, Florence, Hildegarde ou Geneviève. Malgré ces variations, il garde toujours une structure semblable, comme on peut le peut

I. «Cette [chanson] est comment La Sainte Vierge Marie a aidé l’impératrice de Rome à supporter les grandes peines qu'elle a endurées ».

2. Ce qui veut dire: «Et cela je veux vous le conter comme il est écrit » ou bien « Cette chanson se fit d'après ce que la lettre en dit ».

3. Au XIII ${ }^{\mathrm{e}}$ siècle, on peut trouver cette histoire dans: Speculum Historiale (I2I7) de Vincent de Beauvais, Stella maris (I248-9) de John of Garland et Miracles de Nostre Dame (I2I8-I928) de Gautier de Coincy. Et au XV siècle elle est incluse dans Les Miracles de Nostre Dame de Jean de Miélot, dans Miraculorum (Reims I400) et dans Miracles of the Blessed Virgin Mary de Johannes Herolt. 
voir dans les contes folkloriques, plus particulièrement dans l'aire méditerranéenne et dans les pays de la vielle Russie ( $c f$. ATU 88I et 883$).^{4}$

Une autre femme innocente injustement accusée d'adultère est Geneviève, qui apparait dans un récit à motivation religieuse, écrit en latin vers le dernier tiers du XIV ${ }^{\text {ème }}$ siècle, probablement, par un moine du monastère allemand de Sainte Marie de Laach ${ }^{5}$. On attribue ici l'aventure à la fille du Duc de Brabant, nommée Geneviève et mariée avec le comte Sigfride de Ballen, qui part en Croisade et laisse sa femme sous la surveillance de son domestique Golo. Celui-ci lui demande d'établir une relation amoureuse avec lui et devant le refus de Geneviève, il l'accuse d'indifélité auprès de son époux qui ordonne de la tuer dans la forêt. Le récit de Geneviève est rempli de motifs d'origines diverses, légendaires, folkloriques et savantes. Il a été composé à partir d'éléments de la vie de Marie de Brabant, faussement accusée d'adultère au milieu du XIII ${ }^{\text {ème }}$ siècle, de celle de Sainte Geneviève de Paris et de la «Bonne Florence de Rome » (cf. Vanja, EM 5 : IOO5).

Ce récit est considéré comme ayant influencé le livre Die Königin von Frankreich (La vie de la reine de France), édité en I465, bien que pour Mussafia (I866: $669)^{6}$ cette histoire dérive d'un autre récit antérieur et de caractère religieux, très populaire en Allemagne, probablement élaboré à partir des éléments de la vie de Sainte Isabelle de Hongrie, qui a vécu au XIII ${ }^{\text {ème }}$ siècle. Malgré tout, l'origine et le processus de transmission de la légende de Geneviève ne sont pas clairs. À partir du XVI et XVII ${ }^{\text {ème }}$ siècles la légende de ce personnage est transmise en plusieurs copies, dans des collections de récits legendaires, dans des livres hagiographiques et dans des versions orales, et elle devient très populaire. Mais, est-ce également un conte traditionnel?

La cantiga du roi Alphonse $\mathrm{X}$ a dû avoir une influence dans l'élaboration de trois autres histoires ('nouvelles') semblables en castillan, insérées dans un manuscrit du XV ème siècle lui aussi conservé à l'Escorial. Le manuscrit a été transcrit par le professeur américain Thomas D. Spaccarelli, qui l'a titré «Libro de los Huéspedes $"{ }^{8}$ en référence aux pèlerins qui venaient à Compostelle et logeaient dans

4. C'est ainsi que pour Hans-Jörg Uther (EM 3: I67-I7I) le titre «Crescencia » qu'Aarne et Thompson ont assigné au conte-type 7I2, devient trompeur, parce que l'histoire de Crescencia représente seulement une part de la tradition; les contes référés dans AT 7I2 s'adaptent mieux aux types $88 \mathrm{I}$ et $883 \mathrm{~A}$.

5. Les manuscrits les plus anciens qui racontent cette histoire sont datés de I472 et de I50O, copies du Prieur des carmélites, Matias von Eymich, et du moine bénédictin Johanes von Audernach (EM s.v. « Genovefa »)

6. Cité par Espinosa, I947, II: 400-40I.

7. Thomas Spaccarelli est professeur émérite à l'University of the South de Sewanee (Etats Unis d'Amérique). Il est l'éditeur du manuscrit, Text and Concordance of «El libro de los huespedes » (Escorial MS. h.I.I3), publié à Madison par l' Hispanic Seminary of Medieval Studies, en 1996. Il a écrit aussi plusieurs articles en relation avec les récits du manuscrit.

8. Il y a des éditions plus actuelles de l'ensemble des textes qui forment le manuscrit: John Rees-Smith (ed.): The Lives of St Mary Magdalene and St Martha (MS Esc. h-I-I3). Exeter: University of Exeter, 1989; Roger Walker (ed.) : Estoria de Santa María Egiçiaca (MS Escurialense

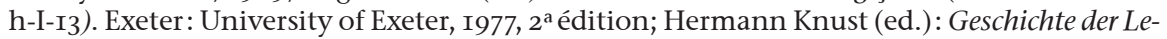
genden der h. Katharina von Alexandrien un der h. Maria Aegyptiaca. Niemeyer: Halle, I890; Roger M. Walker (ed.) : El cavallero Plaçidas (MS Esc. h-I-I3). Exeter: University of Exeter, I982; John R. Maier (ed.) : El Rrey Guillelme. Exeter: University of Exeter, I984; Herbert L. Baird, Jr. 
les monastères, où la lecture à haute voix des récits de ce manuscrit, ainsi que les cantilènes de celui du roi Alphonse X, servaient de divertissement aux pèlerins du Chemin de Saint Jacques.

Voici les longs titres que portent les récits:

I. «El cuento muy fermoso del enperador Otas de Roma y de la infante Florençia su fija y del buen cauallero Esmero $»^{9}$ (folios 48b-99c).

2. Vn muy fermoso cuento de una santa enperatris que ouo en Rroma y de su castidad» (99c-I24b).

3. «Vn noble cuento del enperador Carlos Maynes de Roma y de la buena enperatris Seuilla su mugier» (ff. I24b-I52A).

Dans ces trois récits comme dans la «Cantiga V », l'adjuvant magique est la Vierge Marie, qui dès le XII ${ }^{\text {ème }}$ siècle était un personnage habituel dans les récits hagiographiques en latin; par contre, chacune des héroïnes porte un nom différent: Florence dans le premier, l'impératrice de Rome dans le second et l'impératrice Séville, ${ }^{\text {Io }}$ dans le troisième.

Au milieu du XVI ${ }^{\text {ème }}$ siècle, Francesco Straparola traite aussi ce thème dans la troisième nuit (Fable III) de ses Piacevoli notti (« Les nuits facétieuses »). Il en attribue l'histoire au personnage de Blanchebelle, l'épouse de Ferradin, roi de Naples. Là, c'est la marâtre de Ferradin qui, jalouse, ordonne à ses domestiques de tuer l'héroïne dans la forêt; ils ne la tuent pas mais lui coupent les mains et lui crèvent les yeux avant de l'abandonner. Straparola combine ici des motifs des contestypes 7I2 (Crescentia) et 706 (The Maiden without Hands) pour élaborer un récit vraiment merveilleux, où une couleuvre, sœur de lait de l'héroïne, ensorcelée et possédant des pouvoirs magiques, occupe la place de la Vierge Marie.

Malgré la vitalité de l'histoire de l'impératrice de Rome dès le haut Moyen Âge, diffusée plus tard à travers les pays européens et dans les aires linguistiques de la Péninsule ibérique on ne connaît pas de versions orales. ${ }^{\text {II }}$ Seules circulent à l'époque moderne celles qui racontent l'histoire de Geneviève ${ }^{\mathrm{I2}}$ et celles concernant une innocente jeune fille, accusée de ne pas être restée chaste. La large diffusion de la légende de Geneviève en Europe à partir du XVI ${ }^{\text {ème }}$ siècle, en grande partie, due à l'influence de l'Église qui en a fait une lecture obligatoire pour instruire les jeunes filles dans la chasteté, a donné une telle popularité à cette histoire qu'elle a vraisemblablement éclipsé la longue histoire de Crescencia, plus difficile à mémoriser.

(ed.): Análisis lingüístico y filológico de «Otas de Roma ». Anejos del BRAE, Madrid, I976; Anita Benaim de Lasry (ed.): " Carlos Maynes » and "La enperatris de Roma »: Critical Edition and Study of Two Medieval Spanish Romances. Juan de la Cuesta, Newark, Delaware, I982.

9. 1 . « Le très beau conte de l'empereur Otas de Roma et de l'infante Florençia, sa fille et du bon chevalier Esmero ». 2. «Un très beau conte d'une sainte impératrice qu'il y a eu à Rome et de sa chasteté ». 3. «Un noble conto de l'enpereur Charles Maynes de Roma et de la bonne impératrice Sevilla sa femme ».

Io. 'Sevilla' s'appelle l'héroïne des contes de femme faussement accusée d'infidélité dans les versions espagnoles écrites (l'épouse de l'empereur Charlemagne parfois), depuis les temps les plus reculés (cf. Richard W. Tyler I967).

II. Il y a une version portugaise, « Hestória d'emperatriz », à peu près pareille à celle du roi de Castille, recueillie par María Leonor Carvalhão Buescu (I984: I98-I99) à une dame de Monsanto, mais, il s'agit, sans doute, d'une version savante appris par cour.

I2. Un récit oral tiré, sans doute, de l'histoire fabuleuse de la fille du duc de Brabant.

Estudis de Literatura Oral Popular, núm. 4, 20I5 


\section{Classifications d'ATU}

\subsection{Le type 712 (Crescentia)}

L'ATU a gardé le numéro de conte-type 7I2 de l'Aarne-Thompson pour classer les versions de l'histoire de l'impératrice de Rome, dans le groupe de contes merveilleux, sans doute, du fait des pouvoirs de guérison que l'héroïne reçoit d'un adjuvant surnaturel (la Sainte Vierge Marie). Mais, sous ce numéro de type, l'ATU a rassemblé des versions orales en langue castillane et portugaise de l'histoire de "Geneviève ", à laquelle on ajoute parfois l'adjectif 'sainte' et dont nous avons plusieurs versions aussi en langue galicienne. Ce récit ne comporte pas de motifs magiques ou surnaturels.

On analysera maintenant l'histoire donnée en galicien par la Cantiga 5 des Cantigas de Santa María, écrite à la demande du roi Alphonse X comme nous l'avons dit plus haut. Voilà ce que cette histoire raconte:

L'empereur de Rome part en Croisade et laisse son épouse aux soins de son frère. Celui-ci veut la posséder et elle le fait emprisonner dans une tour. Quand l'impératrice apprend l'arrivée de son mari elle libère son beau-frère, qui l'accuse d'adultère. L'empereur ordonne à ses domestiques d'aller tuer sa femme dans un bois. Avant de la faire disparaître, ils tentent d'abuser d'elle. Un comte la tire d'affaire et la ramène chez lui pour qu'elle s'occupe de son enfant, mais le frère du comte, à travers des épisodes symétriques aux antérieurs, tente de la séduire. Elle refuse, et il se venge, tue l'enfant et l'accuse d'être la meurtrière. La femme, à nouveau condamnée à mort, est confiée à un batelier pour qu'il la jette à la mer. Avant d'exécuter l'ordre de la jeter, il tente de la violer, mais la femme appelle la Vierge et une voix du ciel dit au batelier de s'arrêter; il la dépose sur un rocher. Elle s'endort et rêve qu'elle est sauvée par la Vierge Marie, laquelle lui offre une plante miraculeuse capable de guérir la lèpre. Le mari, sans la reconnaître, appelle son épouse pour qu'elle vienne soigner son beau-frère qui est malade de la lèpre. La femme, qui le reconnaît, lui fait avouer ses péchés devant le Pape et devant son époux, puis elle leur révèle son identité. Son mari lui demande pardon et la supplie de rentrer à la maison avec lui, mais elle s'y refuse et se retire dans un couvent à Paris.

Ce récit, comme l'ensemble des Cantigas de Santa María a pour intention manifeste de favoriser le culte de la Sainte Vierge. C'est grâce à l'intervention de celleci que l'héroïne de la cantiga $\mathrm{V}$ peut se sauver. Il est évident que, comme toute la littérature hagiographique, cette œuvre fut élaborée pour montrer que le pouvoir est dans le divin et dans la religion, c'est-à-dire dans l'Église de Rome et ses serviteurs les rois.

Bien que provenant d'une origine orale, les récits racontant des miracles de la Vierge ou des saints sont donc bien des récits « savants ", non réellement populaires, « et témoignent de la volonté acculturante de ceux qui possèdent le savoir » (Saugnieux I978 : 79). Mais, sont-ils des contes merveilleux? Si cette histoire est classée sous le type 7I2 en raison des pouvoirs de guérison que l'héroïne reçoit de la Vierge, pourquoi ne pas la classer parmi les contes religieux?

Parmi les versions orales du thème de la femme innocente calomniée, nous avons évoqué plus haut celles qui circulent en castillan et galicien dont l'héroïne est Geneviève. Toutes sont rattachées au conte-type 7I2 bien qu'on n'y trouve pas d'éléments magiques, pas d'intervention de la Vierge et pas d'herbes médi- 
cinales non plus. Sous l'influence de l'Église, les versions concernant Geneviève sont racontées avec beaucoup d'effets dramatiques et font ressortir la docilité et la résignation de l'innocente héroïne, enceinte, face aux peines infligées par le traître. Elles insistent sur le vécu de la femme dans la forêt. Il s'agit là d'un épisode qui n'est pas signalé dans la version du roi Alphonse X, même s'il était déjà présent au V vème siècle dans le drame hindou Sakuntalã, selon dit Frenzel (I980: I29). À moins que l'on ne considère la sainteté de la femme fidèle, absolument chaste et bienfaisante, comme un élément surnaturel étant donné les circonstances difficiles qu'elle avait endurées, on ne voit pas la raison pour rattacher ces contes à l'ensemble des merveilleux.

Voici les épisodes intégrés dans nos versions orales:

I. Pendant l'absence de son époux, sa femme, qui est enceinte ou a déjà un enfant, est accusée d'adultère par son beau-frère (un domestique), dont elle a repoussé les avances.

2. En revenant, le mari envoie un ou plusieurs domestiques pour qu'ils la tuent dans le bois, mais ils s'apitoient sur son sort et ne la tuent pas, ils l'abandonnent.

3. Elle vit avec son fils dans une cave en se nourrissant de fruits et du lait qu'elle trait généralement d'une biche.

4. Un jour où le roi va à la chasse il rencontre par hasard sa femme et son fils. Ils se reconnaissent et ils se réconcilient.

5. Le traître est puni de mort ou pardonné par la femme.

Il faut souligner, dans ce récit, la présence d'un adjuvant animal, un cerf (chèvre ou chevrette) qui apporte du lait à l'enfant et des fruits pour la femme. Bien qu'il n'ait pas de pouvoirs magiques explicites, on peut en déduire qu'il est un envoyé divin pour aider la vertueuse et sainte femme. L'une de versions orales galiciennes, «A muller abandonada no monte ${ }^{13}$ » (Noia 2002 : 205-207), dont l'héroïne n'est pas nommée, ajoute le motif de l'enfant sauvage sous la figure d'un des jumeaux mis au monde par l'héroïne et qui s'est égaré dans la forêt.

Malgré les coïncidences avec la version médiévale, il est probable que la plupart des récits oraux procèdent directement d'un roman, écrit au XIX ${ }^{\text {ème }}$ siècle, au moment où l'histoire réapparaît avec un grand succès, dans la tradition du Romantisme allemand. On voit apparaître à ce moment-là de nombreuses adaptations du thème de la femme calomniée sous forme de pièces de théâtre, récits et romans. L'un des romans les plus répandus en Europe fut le Geneviève de Brabant du prêtre catholique Christopher von Schmid (I768-I864), publié en I825 à Augsbourg, qui fut traduit en castillan déjà en I859 (Frenzel I980: I77-I78). Le succès de ce roman a été tel que de nombreuses rééditions en ont été publiées en Espagne durant le XIX ${ }^{\text {ème }}$ et le $\mathrm{XX}^{\text {ème }}$ siècle.

\subsection{Le type $883 A$ (The Innocent Slandered Maiden)}

Le conte-type 883A (L'innocente jeune fille calomniée), est représenté par un grand nombre de versions européennes et arabes ATU (2004). Le Catálogo español de Camarena-Chevalier (I996) mentionne cinq versions orales en castillan. Le ca-

I3. « La femme abandonnée dans la forêt ». 
talogue catalan (Oriol-Pujol 2003) indique une version recueillie à Mallorca et le catalogue portugais de Cardigos (2006) en signale huit versions. On n'en connaît pas de versions écrites de ce type à l'exception d'un conte iranien présent dans le livre Tuti-nama («Les contes du perroquet ») au XIV ${ }^{\text {ème }}$ siècle. Et on n'a pas trouvé ce récit en langue galicienne.

Voici les épisodes des versions espagnoles:

I. En l'absence du père, une jeune fille, reste sous la protection d'un serviteur ou de son oncle (parfois, un curé). Il tente de la séduire et n'y parvenant pas l'accuse de mener une vie dissolue.

2. Le père oblige son fils (un domestique) à l'emmener au bois pour la tuer, mais il ne la tue pas et l'abandonne dans ce bois.

3. Un honnête prince (un capitaine) la retrouve, et elle est prise en charge par cet homme qui la ramène chez lui. La jeune fille va travailler dans une échoppe où elle rencontre un autre personnage de haut rang qui l'épouse.

4. Là apparaît un nouveau méchant calomniateur qui accuse l'héroïne d'adultère. Elle s'enfuit. Si elle a eu un enfant, il le tue et dit au mari que c'est elle qui l'a tué.

5. Alors qu'elle est recueillie dans une maison en tant que domestique, son époux arrive, accompagné du traître.

6. La femme fait venir tous ceux qui l'ont accusée et leur prépare à manger, puis, elle raconte à table tout ce qui s'est passé et se réconcilie avec son mari.

7. Les traîtres reconnaissent leurs fautes, et le récit se termine par le châtiment.

Certaines versions ont recours au motif du déguisement masculin par la femme qui s'enfuit et revient ensuite à la maison. On trouve aussi d'autres variations.

Si on compare ce récit (T. 883A) avec la version orale de « Geneviève » (T. 7I2) en castillan et galicien, on remarque l'adjonction des épisodes 3, 4, 5 et 6, signalés plus haut. Mais, la différence fondamentale se trouve dans le statut et la caractérisation de l'héroïne. À l'épouse souffrante et passive d'un seigneur (Geneviève) s'oppose une jeune fille célibataire qui, d'une ou d'autre façon, cherche la solution à ses problèmes.

À propos du type 883A, Christine Shojaei Kawan (I996, EM 8, col. I4O2-I4O7, a signalé que:

Conte-nouvelle à deux épisodes qui représente une des multiples variations sur le thème de l'innocence persécutée... L'héroïne cependant ne subit pas son sort en silence, au contraire sa réaction aux dangers est rapide et courageuse... une deuxième tentative de séduction est souvent liée au meurtre des enfants...

Des ressemblances existent, particulièrement avec les conte-types 7I2 (Crescentia) et 88I (Oft-proved Fidelity)... Des critères par lesquels AaTh 883A se distingue de AaTh 7I2 et AaTh 88I sont: premièrement la sousdivision de l'action en un temps avant et un après le mariage, où dans le premier épisode c'est le statut de l'héroïne en tant que fille vertueuse, 
dans le second son honneur d'épouse qui est en jeu, alors que dans AaTh 7I2 et 88I il s'agit uniquement de la confirmation de la fidélité conjugale; en second lieu le fait que la combinaison spécifique des motifs constitutifs s'avère comme un tout très stable. AaTh 7I2 se distingue d'autre part de 883A par sa coloration nettement religieuse.

Si on compare les récits orales espagnols du conte-type 883A avec la cantiga du roi Alphonse X (T. ATU 7I2) les ressemblances sont, par contre, énormes; les deux histoires comportent des épisodes pareils et se déroulent de la même façon, surtout dans la première partie. Dans la seconde, des motifs de la cantiga se retrouvent aussi dans les contes orales, à l'exception d'éléments religieux (merveilleux) et de la condition de l'héroïne, coïncidant avec l'histoire de Geneviève.

\section{Conclusions}

Pour conclure, on peut dire qu'il semble évident que les deux contes-types racontent la même histoire, et que les récits qu'on vient d'analyser représentent trois des multiples variations sur le thème de l'innocence persécutée. Ce sont des hypertextes dérivés d'un hypotexte peut-être basé sur une histoire réelle (celle de «Crescencia»?), d'où surgirait une légende devenue épopée ainsi que des récits écrits qui circulaient aussi oralement. De nouveau, le thème serait passé de l'oral à l'écrit avec des héroïnes au statut et aux caractéristiques différentes. Entre le texte écrit et le conte oral on n'a cessé de croiser des motifs.

Le fait que n'importe quel conteur ou institution infléchisse ou non l'histoire vers le religieux ne veut pas dire qu'un tel récit quitte le domaine du merveilleux. Dans le cas du conte-type 7I2, comme dans bien d'autres, il semble évident qu'y a eu manipulation consciente afin de consolider le culte marial.

C'est pourquoi délimiter l'appartenance d'un conte au Magic en fonction de la présence des miracles ou d'un personnage qui, à cause de la protection divine, reçoit des pouvoirs surnaturels c'est ne tenir compte ni de la spontanéité de la narration orale ni des croyances du peuple. De la même façon que l'absence d'éléments magiques qui caractérise les « Realistic tales » n'implique pas toujours que les contes inclus dans ce groupe soient réellement réalistes.

Plutôt que l'absence d'éléments surnaturels, la différence fondamentale des versions du type 883A par rapport à celles du type 7I2 est, à mon avis, la présence d'une héroïne courageuse qui prend la décision d'échapper à ses persécuteurs, celle qui pousse à la réunion finale où tout est dévoilé. À l'héroïne patiente décrite à l'époque médiévale dans les textes savants, les conteurs et conteuses populaires opposent la figure d'une femme entreprenante, qui, comme le montre Christine Shojaei Kawan (I996: I705), ne subit pas son sort en silence mais sait réagir avec intelligence et rapidité devant les problèmes. Bien que, ajoute-t-elle, «le récit ne peut être décrit comme un conte féminin que selon sa structure de surface. Des valeurs patriarcales, dont le père apparaît comme le garant, sont au centre de l'action. "

Josiane Bru (2OI4) arrive à la même conclusion dans un article sur le conte «Barbe Bleue » où elle compare les motifs et les héroïnes de versions orales et écrites différentes. Bru souligne que l'héroïne de la version de Perrault est une femme passive et oisive sous la férule du pouvoir patriarcal, «immature et futile», 
au contraire de l'héroïne populaire entreprenante et hardie des contes oraux. « Elle participe, d'une ou l'autre façon, à son propre sauvetage » /.../ en prévenant " elle-même ses parents en leur envoyant un message par son animal de compagnie ou encore en criant par la fenêtre pour alerter ses frères ", dit Bru (2OI4: I4OI4I)

Bien que -et voilà nôtre tâche- pour pouvoir arriver à une classification sur des bases plus solides, il serait nécessaire d'étudier la composition de chaque récit dans une région particulière. La caractérisation de contes-nouvelles à partir d'une approche textuelle apporterait une meilleure délimitation du groupe. En plus du thème, la caractérisation des héroïnes et les ressources stylistiques pourraient se révéler essentielles pour déterminer l'attribution de contes au groupe de « Realistic Tales », en général, peu nombreux dans les catalogues régionaux ou de «Magic Tales».

\section{Références bibliographiques}

AARne, Antti ; Stith Thompson (I98I): The Types of the Folktale. A Classification and Bibliography. Folklore Fellows' Comunications I84. Helsinki: Suomalainen Tiedeakatemia. Première édition en I928 et seconde en I96I.

ASIÁIN ANSORENA, Alfredo (2006) : Narraciones folclóricas navarras. Recopilación, clasificación y análisis. Cuadernos de Etnología y Etnografía de Navarra no 8I. Navarra: Gobierno de Navarra.

ATU (2004) : UTHER, Hans-Jörg : The Types of International Folktales. Folklore Fellows' Communications 284, 285, 286. Helsinki : Suomalainen Tiedeakatemia. Trois volumes.

BARANDiARán, José Miguel de (I960-62) : El mundo y la mente popular vasca. San Sebastián: Auñamendi. Quatre volumes.

BRAgA, Theófilo (I9I4): Contos tradicionais do povo portugués. Lisboa: Rodríguez. Deux volumes.

BRU, Josiane (2OI4) : «Du sire de Carnoët à Barbe Bleue. Le conte, la légende, le mythe ». Dans Yves CoATIvy et Fanch Postic (dir.): La forêt de Carnoët (Quimperlé). Archéologie, histoire, traditions et légendes, Brest et Quimperlé : Société d'Histoire du Pays de Kemperle et Centre de Recherche Bretonne et Celtique, p. I3I-I5I.

Camarena, Julio; Maxime Chevalier (2003) : Catálogo tipológico del cuento folklórico español. Cuentos-novela. Madrid: Centro de Estudios cervantinos.

CARDigos, Isabel (2006): Catalogue of Portuguese Folktales. Helsinki: Suomalainen Tiedeakatemia.

CARVAlHÃo BUESCU, Ma Leonor (I984): Monsanto. Etnografía e Linguagem. Lisboa: Presença, seconde édition, p. I98-I99.

CoINCY, Gautier (2007) : Cinq Miracles de Notre-Dame. Traduction, introduction et notes de Jean-Louis GABRIEL BENOIT. Collection «Classiques français du Moyen Âge ». Paris: Honoré Champion.

Curiel Merchán, Marciano (2006) : Cuentos extremeños. Mérida : Editora regional de Extremadura. Première édition en I944. 
Delarue, Paul ; Marie-Louise TÉnÈze (2000) : Le conte populaire français: ContesNouvelles. Avec la collaboration de Josiane BRU. Paris : Éditions du C.T.H.S.

DURÁN, Agustín (I945): Romancero General o la Colección de romances castellanos anteriores al siglo XVIII. Madrid: B.A.E. et Atlas, X et XVI, deux volumes.

EM: Enzyklopädie des Märchens. Handwörterbuch zur historischen und vergleichenden Erzählforschung. Begründet von Kurt Ranke, ed. Rolf Wilhelm BREDNICH u.a. Berlin/New York: Walter de Gruyter. Dès I977.

EsPINOSA, Aurelio (I946-47) : Cuentos populares españoles. Recogidos de la tradición oral de España. Madrid: CSIC.

FRENZEL, Elisabeth (I980): Diccionario de motivos de la literatura universal. Madrid: Gredos. Première édition en I976.

MussafiA, Adolf (I866) : « Über eine italienische metrische Darstellung der Chrescentiasage ». Sitzunsberichte $\mathrm{n}^{\circ}$ 51: 589-692.

NoiA CAMpos, Camiño (2002): Contos galegos de tradición oral. Vigo : Nigratrea. Traductión castillane en 2003: Cuentos gallegos de tradicción oral. Vigo : Nigratrea.

- (20IO) : Catálogo tipolóxico do conto galego de tradición oral. Vigo: Servizo de Publicacións da Universidade de Vigo.

OrIOL, Carme; Josep M. PUJOL (2003) : Índex tipològic de la rondalla catalana. Barcelona: Generalitat de Catalunya.

Pinto-CorreiA, J. David (I992) : «Para uma teoria do texto da Literatura Popular Tradicional». Dans Manuel Viegas GuerReiro (coord.): Literatura Popular Portuguesa. Teoría da Literatura Oral/Tradicional/Popular. Lisboa : Acarte, Fundação Caluste Gulbenkian (Compilação de comunicações apresentadas no Colóquio de novembro de I987).

SHOJAEI KaWAN, Christine (I996): «Mädchen ». EM 8: I4O2-I707.

SPACCARELli, Thomas (ed.) (I996) : Text and concordance of «El Libro de los Huéspedes» (Escorial ms. h.I.I3). Madison: Hispanic Seminary of Medieval Studies.

SAUGNiEuX, Joël (I978) : "Culture populaire et culture savante dans l'oeuvre de Berceo (problèmes de méthode) ». Dans Actas de las II Jornadas de Estudios Berceanos. Berceo no 94-95: 65-84.

ThOMPSON, Stith (1955-58) : Motif-lndex of Folk Literature: a classification of narrative elements in folktales, ballads, miths, fables, mediaeval romances, exempla, fabliaux, jest-books and local legends. Six volumes. $2^{\mathrm{a}} \mathrm{ed}$. Copenhagen and Bloomington: Indiana Univ. Press.

Tyler, Richard W. (I967): «Algunas versiones de la leyenda de la Reina Sevilla en la primera mitad del Siglo de Oro ». Dans Norbert Polussen; Jaime SánChez Romeralo (coords.) : Actas del Segundo Congreso Internacional de Hispanistas, célebré à Nimega en I965. Nimega : Instituto Español de la Universidad de Nimega. <http://cvc.cervantes.es/literatura/aih/pdf/o2/aih_O2_I_o64.pdf>

UTHER, Hans-Jörg (I98I): «Crescentia ». EM 3: I67-I7I.

VANJA, Konrad (I987): «Genovefa ». EM 5: I003-Ioo9. 